\title{
Oscillation Criteria of Second-Order Dynamic Equations with Damping on Time Scales
}

\author{
Yang-Cong Qiu ${ }^{1}$ and Qi-Ru Wang ${ }^{2}$ \\ ${ }^{1}$ School of Humanities \& Social Science, Shunde Polytechnic, Foshan, Guangdong 528333, China \\ ${ }^{2}$ School of Mathematics \& Computational Science, Sun Yat-Sen University, Guangzhou, Guangdong 510275, China
}

Correspondence should be addressed to Qi-Ru Wang; mcswqr@mail.sysu.edu.cn

Received 11 December 2013; Accepted 19 January 2014; Published 17 March 2014

Academic Editor: Ferhan M. Atici

Copyright (C) 2014 Y.-C. Qiu and Q.-R. Wang. This is an open access article distributed under the Creative Commons Attribution License, which permits unrestricted use, distribution, and reproduction in any medium, provided the original work is properly cited.

Using functions in some function classes and a generalized Riccati technique, we establish Kamenev-type oscillation criteria for second-order dynamic equations with damping on time scales of the form $\left(r(t)\left(x^{\Delta}(t)\right)^{\gamma}\right)^{\Delta}+p(t)\left(x^{\Delta}(t)^{\gamma}\right)+f(t, x(g(t)))=0$. Two examples are included to show the significance of the results.

\section{Introduction}

In this paper, we study the second-order dynamic equation with damping

$$
\left(r(t)\left(x^{\Delta}(t)\right)^{\gamma}\right)^{\Delta}+p(t)\left(x^{\Delta}(t)\right)^{\gamma}+f(t, x(g(t)))=0
$$

on a time scale $\mathbb{T}$ satisfying inf $\mathbb{T}=t_{0}$ and sup $\mathbb{T}=\infty$.

Throughout this paper we will assume that

(C1) $r \in C_{\mathrm{rd}}(\mathbb{T},(0, \infty))$;

(C2) $p \in C_{\text {rd }}\left(\mathbb{T}, \mathbb{R}_{+}\right)$, where $\mathbb{R}_{+}:=[0, \infty)$;

(C3) $\gamma$ is a quotient of odd positive integers;

(C4) $g \in \dot{C}(\mathbb{T}, \mathbb{T})$ is nondecreasing and $g(t) \geq t$ for $t \in \mathbb{T}$;

(C5) $f \in C(\mathbb{T} \times \mathbb{R}, \mathbb{R})$ and there exists a function $q \in$ $C_{\mathrm{rd}}\left(\mathbb{T}, \mathbb{R}_{+}\right)$such that $u f(t, u) \geq q(t) u^{\gamma+1}$;

(C6) $-p(t) / r(t)$ is positively regressive, which means $1-$ $\mu(t) p(t) / r(t)>0$ and

$$
\int_{t_{0}}^{\infty}\left(\frac{e_{-p / r}\left(t, t_{0}\right)}{r(t)}\right)^{1 / \gamma} \Delta t=\infty .
$$

The theory of time scales, which has recently received a lot of attention, was introduced by Hilger in his Ph.D. thesis [1] in 1988 in order to unify continuous and discrete analysis; see also [2]. Preliminaries about time scale calculus can be found in [3-6] and hence we omit them here. Note that, for some typical time scales, we have the following properties, respectively:

(1) since $\mathbb{T}=\mathbb{R}_{+}$, we have

$$
\begin{gathered}
\sigma(t)=\rho(t)=t, \quad f^{\Delta}(t)=f^{\prime}(t), \\
\int_{a}^{b} f(t) \Delta t=\int_{a}^{b} f(t) d t ;
\end{gathered}
$$

(2) since $\mathbb{T}=\mathbb{N}_{0}$, we have

$$
\begin{gathered}
\sigma(t)=t+1, \quad \rho(t)=t-1, \\
f^{\Delta}(t)=f(t+1)-f(t), \\
\int_{a}^{b} f(t) \Delta t=\sum_{k=a}^{b-1} f(k), \quad a \leq b ;
\end{gathered}
$$


(3) since $\mathbb{T}=\left\{2^{n}, n \in \mathbb{N}_{0}\right\}$, we have

$$
\begin{gathered}
\sigma(t)=2 t, \quad \rho(t)=\frac{t}{2}, \quad f^{\Delta}(t)=\frac{f(2 t)-f(t)}{t}, \\
\int_{a}^{b} f(t) \Delta t=\sum_{k=\log _{2} a}^{\log _{2} b-1} f\left(2^{k}\right) 2^{k}, \quad a \leq b .
\end{gathered}
$$

Definition 1. A solution $x$ of (1) is said to have a generalized zero at $t^{*} \in \mathbb{T}$ if $x\left(t^{*}\right) x\left(\sigma\left(t^{*}\right)\right) \leq 0$ and it is said to be nonoscillatory on $\mathbb{T}$ if there exists $t_{0} \in \mathbb{T}$ such that $x(t) x(\sigma(t))>0$ for all $t>t_{0}$. Otherwise, it is oscillatory. Equation (1) is said to be oscillatory if all solutions of (1) are oscillatory.

In recent years, there has been much research activity concerning the oscillation and nonoscillation of solutions of dynamic equations on time scales; for example, see [1-16] and the references therein. In Došlý and Hilger [9], the authors considered the second-order dynamic equation as

$$
\left(p(t) x^{\Delta}(t)\right)^{\Delta}+q(t) x(\sigma(t))=0
$$

and gave necessary and sufficient conditions for the oscillation of all solutions on unbounded time scales. In Del Medico and Kong [7, 8], the authors employed the following Riccati transformation:

$$
u(t)=\frac{p(t) x^{\Delta}(t)}{x(t)}
$$

and gave sufficient conditions for Kamenev-type oscillation criteria of (6) on a measure chain.

In Wang [15], the author considered second-order nonlinear damped differential equation as

$$
\begin{array}{r}
\left(a(t) \psi(x(t)) k\left(x^{\prime}(t)\right)\right)^{\prime}+p(t) k\left(x^{\prime}(t)\right)+q(t) f(x(t))=0, \\
t \geq t_{0},
\end{array}
$$

using the following generalized Riccati transformations:

$$
\begin{aligned}
& v(t)=\phi(t) a(t)\left[\frac{\psi(x(t)) k\left(x^{\prime}(t)\right)}{f(x(t))}+R(t)\right], \quad t \geq t_{0}, \\
& v(t)=\phi(t) a(t)\left[\frac{\psi(x(t)) k\left(x^{\prime}(t)\right)}{x(t)}+R(t)\right], \quad t \geq t_{0},
\end{aligned}
$$

where $\phi \in C^{1}\left(\left[t_{0}, \infty\right), \mathbb{R}_{+}\right), R \in C\left(\left[t_{0}, \infty\right), \mathbb{R}\right)$, and gave new oscillation criteria of (8). In [12], Saker considered secondorder nonlinear neutral delay dynamic equation as

$$
\left(r(t)\left((y(t)+p(t) y(t-\tau))^{\Delta}\right)^{\gamma}\right)^{\Delta}+f(t, y(t-\delta))=0
$$

and improved some well-known oscillation results for second-order neutral delay difference equations. In [13], Saker et al. studied the second-order damped dynamic equation with damping as follows:

$$
\left(a(t) x^{\Delta}(t)\right)^{\Delta}+p(t) x^{\Delta^{\sigma}}(t)+q(t)\left(f \circ x^{\sigma}\right)=0
$$

and gave some new oscillation criteria. In Huang and Wang [10], the authors considered second-order nonlinear dynamic equation as

$$
\left(p(t) x^{\Delta}(t)\right)^{\Delta}+f(t, x(\sigma(t)))=0 .
$$

By using a similar generalized Riccati transformation which is more general than (7),

$$
u(t)=\frac{A(t) p(t) x^{\Delta}(t)}{x(t)}+B(t),
$$

where $A \in C_{\mathrm{rd}}^{1}\left(\mathbb{T}, \mathbb{R}_{+} \backslash\{0\}\right), B \in C_{\mathrm{rd}}^{1}(\mathbb{T}, \mathbb{R})$, the authors extended the results in Del Medico and Kong [7, 8] and established some new Kamenev-type oscillation criteria. In [11], Qiu and Wang considered the second-order nonlinear dynamic equation of a more general form

$$
\left(p(t) \psi(x(t)) k \circ x^{\Delta}(t)\right)^{\Delta}+f(t, x(\sigma(t)))=0
$$

and established some Kamenev-type oscillation criteria.

In [14], Şenel had tried to establish Kamenev-type oscillation criteria for (1). However, it seemed that several mistakes had been made and the obtained theorems and corollaries are incorrect. In this paper, we will correct some mistakes in [14] and establish some Kamenev-type oscillation criteria for (1) by employing functions in some function classes and a similar generalized Riccati transformation as (13) and as used in $[15,16]$ for nonlinear differential equations. Finally, two examples are included to show the significance of the results.

For simplicity, throughout this paper, we denote $(a, b) \cap$ $\mathbb{T}=(a, b)_{\mathbb{T}}$, where $a, b \in \mathbb{R}$, and $[a, b]_{\mathbb{T}},[a, b)_{\mathbb{T}},(a, b]_{\mathbb{T}}$ are denoted similarly.

\section{Preliminary Results}

To establish Kamenev-type criteria for oscillation of (1), we give three lemmas in this section.

Lemma 2. Assume that (C1)-(C6) hold and there exists a sufficiently large $t_{1} \in\left[t_{0}, \infty\right)_{\mathbb{T}}$ such that $x(t)$ is a solution of (1) satisfying $x(t)>0$ for $t \in\left[t_{1}, \infty\right)_{\mathbb{T}}$. Then, for $t \in\left[t_{1}, \infty\right)_{\mathbb{T}}$, one has

$$
\begin{gathered}
{\left[\frac{r(t)\left(x^{\Delta}(t)\right)^{\gamma}}{e_{-p / r}\left(t, t_{1}\right)}\right]^{\Delta}<0, \quad x^{\Delta}(t)>0,} \\
\left(r(t)\left(x^{\Delta}(t)\right)^{\gamma}\right)^{\Delta}<0 .
\end{gathered}
$$

Proof. Let $t_{1} \in\left[t_{0}, \infty\right)_{\mathbb{W}}$ such that $x(t)$ is a solution of $(1)$ satisfying $x(t)>0$ for $t \in\left[t_{1}, \infty\right)_{\mathbb{T}}$; then we also have $x(g(t))>0$. By (1) and (C5), it follows that, for $t \in\left[t_{1}, \infty\right)_{\mathbb{T}}$,

$$
\left(r(t)\left(x^{\Delta}(t)\right)^{\gamma}\right)^{\Delta}+p(t)\left(x^{\Delta}(t)\right)^{\gamma}=-f(t, x(g(t)))<0,
$$


so we have

$$
\begin{aligned}
& {\left[\frac{r(t)\left(x^{\Delta}(t)\right)^{\gamma}}{e_{-p / r}\left(t, t_{1}\right)}\right]^{\Delta}} \\
& \quad=\frac{\left(r(t)\left(x^{\Delta}(t)\right)^{\gamma}\right)^{\Delta} e_{-p / r}\left(t, t_{1}\right)+p(t)\left(x^{\Delta}(t)\right)^{\gamma} e_{-p / r}\left(t, t_{1}\right)}{e_{-p / r}\left(t, t_{1}\right) e_{-p / r}\left(\sigma(t), t_{1}\right)} \\
& \quad<0 .
\end{aligned}
$$

Assume that there exists $t_{2} \geq t_{1}$ satisfying $x^{\Delta}\left(t_{2}\right)<0$; then, for $t \in\left[t_{2}, \infty\right)_{\mathbb{T}}$, we also have

$$
\left[\frac{r(t)\left(x^{\Delta}(t)\right)^{\gamma}}{e_{-p / r}\left(t, t_{2}\right)}\right]^{\Delta}<0 .
$$

Integrating (18) from $t_{2}$ to $t \in\left[t_{2}, \infty\right)_{\mathbb{T}}$, we obtain

$$
\begin{aligned}
& \frac{r(t)\left(x^{\Delta}(t)\right)^{\gamma}}{e_{-p / r}\left(t, t_{2}\right)}-\frac{r\left(t_{2}\right)\left(x^{\Delta}\left(t_{2}\right)\right)^{\gamma}}{e_{-p / r}\left(t_{2}, t_{2}\right)} \\
& =\frac{r(t)\left(x^{\Delta}(t)\right)^{\gamma}}{e_{-p / r}\left(t, t_{2}\right)}-r\left(t_{2}\right)\left(x^{\Delta}\left(t_{2}\right)\right)^{\gamma}<0
\end{aligned}
$$

that is,

$$
r(t)\left(x^{\Delta}(t)\right)^{\gamma}<r\left(t_{2}\right)\left(x^{\Delta}\left(t_{2}\right)\right)^{\gamma} e_{-p / r}\left(t, t_{2}\right),
$$

which implies that

$$
x^{\Delta}(t)<r^{1 / \gamma}\left(t_{2}\right) x^{\Delta}\left(t_{2}\right)\left(\frac{e_{-p / r}\left(t, t_{2}\right)}{r(t)}\right)^{1 / \gamma} .
$$

Integrating (21) from $t_{2}$ to $t \in\left[t_{2}, \infty\right)_{\mathbb{T}}$ and letting $t \rightarrow \infty$, by (C6), we obtain

$$
\begin{aligned}
x(t)< & x\left(t_{2}\right) \\
& \quad+r^{1 / \gamma}\left(t_{2}\right) x^{\Delta}\left(t_{2}\right) \int_{t_{2}}^{t}\left(\frac{e_{-p / r}\left(s, t_{2}\right)}{r(s)}\right)^{1 / \gamma} \Delta s \longrightarrow-\infty,
\end{aligned}
$$

which contradicts $x(t)>0$. Hence $x^{\Delta}(t)>0$, for $t \in$ $\left[t_{1}, \infty\right)_{\mathbb{T}}$, which implies from (16) that $\left(r(t)\left(x^{\Delta}(t)\right)^{\gamma}\right)^{\Delta}<0$ holds. Lemma 2 is proved.

Remark 3. In $\left[14,\left(\mathrm{~A}^{*}\right)\right]$, the key condition that $-p(t) / r(t)$ is regressive is missed; then the assumption

$$
\int_{t_{0}}^{\infty}\left(\frac{e_{-p / r}\left(t, t_{0}\right)}{r(t)}\right)^{1 / \gamma} \Delta t=\infty
$$

may not be well presented. In this paper, the condition is added as (C6).
Remark 4. In $[14,(2.5)]$, it seems not to be so obvious to obtain the inequality

$$
y(t)>y\left(t_{2}\right) e_{-p / r}\left(\cdot, t_{2}\right) .
$$

And in $[14,(2.7),(2.8)]$, the symbol $\leq$ should be $<$. We have improved the proof in Lemma 2.

Lemma 5. Assume that (C1)-(C6) hold and $x(t)$ is a solution of (1), $x(t)>0$ for $t \in\left[t_{1}, \infty\right)_{\mathbb{T}}$ with $t_{1} \in\left[t_{0}, \infty\right)_{\mathbb{T}}$. Then for $t \in\left[t_{1}, \infty\right)_{\mathbb{T}}$, if $0<\gamma<1$, one has

$$
\left(\frac{x^{\Delta}(t)}{x^{\sigma}(t)}\right)^{1-\gamma} \geq \alpha_{1}(t)
$$

And, if $\gamma \geq 1$, one has

$$
\left(\frac{x(t)}{x^{\Delta}(t)}\right)^{\gamma-1} \geq \alpha_{2}(t)
$$

where

$$
\begin{aligned}
& \alpha_{1}(t)=\left(\frac{1}{r(t)} \int_{t}^{\infty} q(s) \Delta s\right)^{(1-\gamma) / \gamma}, \\
& \alpha_{2}(t)=\left(r^{1 / \gamma}(t) \int_{t_{1}}^{t} \frac{1}{r^{1 / \gamma}(s)} \Delta s\right)^{\gamma-1} .
\end{aligned}
$$

Proof. Since $x(t)$ is a solution of (1) satisfying $x(t)>0$ for $t \in\left[t_{1}, \infty\right)_{\mathbb{T}}$ with $t_{1} \in\left[t_{0}, \infty\right)_{\mathbb{T}}$, by Lemma 2 , we have

$$
x^{\Delta}(t)>0, \quad\left(r(t)\left(x^{\Delta}(t)\right)^{\gamma}\right)^{\Delta}<0, \quad t \in\left[t_{1}, \infty\right)_{\mathbb{T}} .
$$

From (16) it follows that

$$
\left(r(t)\left(x^{\Delta}(t)\right)^{\gamma}\right)^{\Delta}<-f(t, x(g(t))) .
$$

Integrating (29), we obtain

$$
\begin{aligned}
r(t)\left(x^{\Delta}(t)\right)^{\gamma} & \geq \int_{t}^{\infty} f(s, x(g(s))) \Delta s \\
& \geq \int_{t}^{\infty} q(s) x^{\gamma}(g(s)) \Delta s \\
& \geq x^{\gamma}(g(t)) \int_{t}^{\infty} q(s) \Delta s \\
& \geq\left(x^{\sigma}(t)\right)^{\gamma} \int_{t}^{\infty} q(s) \Delta s \\
& \geq x^{\gamma}(t) \int_{t}^{\infty} q(s) \Delta s .
\end{aligned}
$$

Hence, when $0<\gamma<1$, we have

$$
\left(\frac{x^{\Delta}(t)}{x(t)}\right)^{1-\gamma} \geq\left(\frac{x^{\Delta}(t)}{x^{\sigma}(t)}\right)^{1-\gamma} \geq \alpha_{1}(t), \quad t \geq t_{1} .
$$


Since $\left(r(t)\left(x^{\Delta}(t)\right)^{\gamma}\right)^{\Delta}<0, t \in\left[t_{1}, \infty\right)_{\mathbb{T}}$, for $\gamma \geq 1$, we get

$$
\begin{aligned}
x(t) \geq x(t)-x\left(t_{1}\right) & =\int_{t_{1}}^{t} \frac{\left(r(s)\left(x^{\Delta}(s)\right)^{\gamma}\right)^{1 / \gamma}}{r^{1 / \gamma}(s)} \Delta s \\
& \geq\left(r(t)\left(x^{\Delta}(t)\right)^{\gamma}\right)^{1 / \gamma} \int_{t_{1}}^{t} \frac{1}{r^{1 / \gamma}(s)} \Delta s,
\end{aligned}
$$

and we obtain

$$
\left(\frac{x(t)}{x^{\Delta}(t)}\right)^{\gamma-1} \geq \alpha_{2}(t), \quad t \geq t_{1} .
$$

So Lemma 5 is proved.

Remark 6. In [14, (2.9), (2.10)], when $0<\gamma<1$ and for any $t \in$ $\left[t_{1}, \infty\right)_{\mathbb{T}}$, the integral $\int_{t}^{\infty} q(s) \Delta s$ in $\alpha_{1}(t)$ must be convergent, which means that

$$
\int_{t}^{\infty} q(s) \Delta s<\infty .
$$

The condition should be added to the paper.

Lemma 7. Assume that (C1)-(C6) hold and $x(t)$ is a solution of (1) satisfying $x(t)>0$ for $t \in\left[t_{1}, \infty\right)_{\mathbb{T}}$ with $t_{1} \in\left[t_{0}, \infty\right)_{\mathbb{T}}$. For $t \in\left[t_{1}, \infty\right)_{\mathbb{T}}$, define

$$
u(t)=A(t) \frac{r(t)\left(x^{\Delta}(t)\right)^{\gamma}}{x^{\gamma}(t)}+B(t),
$$

where $A \in C_{\mathrm{rd}}^{1}\left(\mathbb{T}, \mathbb{R}_{+} \backslash\{0\}\right), B \in C_{\mathrm{rd}}^{1}(\mathbb{T}, \mathbb{R})$. Then $u(t)$ satisfies

$$
u^{\Delta}(t)+A(t) q(t)-B^{\Delta}(t)-\Phi_{0}(t) \leq 0,
$$

where

$$
\Phi_{0}(t)=\left\{\begin{aligned}
- & A(t) p(t)\left(\alpha_{1}(t)\right)^{\gamma /(1-\gamma)} \\
& +A^{\Delta}(t)\left(\frac{u(t)-B(t)}{A(t)}\right)^{\sigma}-\frac{\gamma A(t) \alpha_{1}(t)}{r(t)} \\
& \times\left[\left(\frac{u(t)-B(t)}{A(t)}\right)^{\sigma}\right]^{2}, \quad 0<\gamma<1, \\
- & A(t) p(t)\left(\frac{\alpha_{2}(t)}{r(t)}\right)^{\gamma}\left[\left(\frac{u(t)-B(t)}{A(t)}\right)^{\sigma}\right]^{\gamma} \\
& +A^{\Delta}(t)\left(\frac{u(t)-B(t)}{A(t)}\right)^{\sigma}-\frac{\gamma A(t) \alpha_{2}(t)}{r(t)} \\
& \times\left[\left(\frac{u(t)-B(t)}{A(t)}\right)^{\sigma}\right]^{2}, \quad \gamma \geq 1 .
\end{aligned}\right.
$$

Proof. Without loss of generality we may assume that there exists $t_{1} \in\left[t_{0}, \infty\right)_{\mathbb{T}}$ such that $x(t)>0$ for $t \in\left[t_{1}, \infty\right)_{\mathbb{T}}$; then Lemmas 2 and 5 hold. Let $u(t)$ be defined by (35). Then, differentiating (35) and using (1), it follows that

$$
\begin{aligned}
u^{\Delta}= & \left(\frac{A}{x^{\gamma}}\right)\left(r\left(x^{\Delta}\right)^{\gamma}\right)^{\Delta}+\left(\frac{A}{x^{\gamma}}\right)^{\Delta}\left(r\left(x^{\Delta}\right)^{\gamma}\right)^{\sigma}+B^{\Delta} \\
= & \left(\frac{A}{x^{\gamma}}\right)\left(-p\left(x^{\Delta}\right)^{\gamma}-f(t, x(g(t)))\right) \\
& +\frac{A^{\Delta} x^{\gamma}-A\left(x^{\gamma}\right)^{\Delta}}{x^{\gamma}\left(x^{\gamma}\right)^{\sigma}}\left(r\left(x^{\Delta}\right)^{\gamma}\right)^{\sigma}+B^{\Delta} .
\end{aligned}
$$

Using the fact that $f(t, x(g(t))) \geq q(t) x^{\gamma}(g(t)) \geq q(t) x^{\gamma}(t)$, we obtain

$$
\begin{aligned}
u^{\Delta} \leq & -A q+B^{\Delta}-A p\left(\frac{x^{\Delta}}{x}\right)^{\gamma}+A^{\Delta}\left(\frac{r\left(x^{\Delta}\right)^{\gamma}}{x^{\gamma}}\right)^{\sigma} \\
& -A \frac{\left(x^{\gamma}\right)^{\Delta}}{x^{\gamma}}\left(\frac{r\left(x^{\Delta}\right)^{\gamma}}{x^{\gamma}}\right)^{\sigma} .
\end{aligned}
$$

When $0<\gamma<1$, using the Pötzsche chain rule, we have

$$
\left(x^{\gamma}\right)^{\Delta}=\gamma \int_{0}^{1}\left(x+h \mu x^{\Delta}\right)^{\gamma-1} d h \cdot x^{\Delta} \geq \gamma\left(x^{\sigma}\right)^{\gamma-1} x^{\Delta},
$$

and it follows that

$$
\frac{\left(x^{\gamma}\right)^{\Delta}}{x^{\gamma}} \geq \frac{\gamma\left(x^{\sigma}\right)^{\gamma-1} x^{\Delta}}{x^{\gamma}}=\gamma \frac{x^{\Delta}}{x^{\sigma}}\left(\frac{x^{\sigma}}{x}\right)^{\gamma} .
$$

By Lemmas 2 and 5 , for $t \geq t_{1}$, we obtain

$$
\begin{aligned}
\frac{x^{\Delta}}{x^{\sigma}}=\frac{1}{r} \frac{r\left(x^{\Delta}\right)^{\gamma}}{\left(x^{\sigma}\right)^{\gamma}}\left(\frac{x^{\Delta}}{x^{\sigma}}\right)^{1-\gamma} \geq \frac{\alpha_{1}}{r} \frac{\left(r\left(x^{\Delta}\right)^{\gamma}\right)^{\sigma}}{\left(x^{\gamma}\right)^{\sigma}}=\frac{\alpha_{1}}{r}\left(\frac{u-B}{A}\right)^{\sigma}, \\
\frac{x^{\sigma}}{x} \geq 1 .
\end{aligned}
$$

So (39) becomes

$$
\begin{aligned}
u^{\Delta} \leq & -A q+B^{\Delta}-A p\left(\alpha_{1}\right)^{\gamma /(1-\gamma)} \\
& +A^{\Delta}\left(\frac{u-B}{A}\right)^{\sigma}-\frac{\gamma A \alpha_{1}}{r}\left[\left(\frac{u-B}{A}\right)^{\sigma}\right]^{2} .
\end{aligned}
$$

When $\gamma \geq 1$, we have

$$
\left(x^{\gamma}\right)^{\Delta}=\gamma \int_{0}^{1}\left(x+h \mu x^{\Delta}\right)^{\gamma-1} d h \cdot x^{\Delta} \geq \gamma x^{\gamma-1} x^{\Delta},
$$

and it follows that

$$
\frac{\left(x^{\gamma}\right)^{\Delta}}{x^{\gamma}} \geq \frac{\gamma x^{\gamma-1} x^{\Delta}}{x^{\gamma}}=\frac{\gamma x^{\Delta}}{x} .
$$

By Lemmas 2 and 5 , for $t \geq t_{1}$, we obtain

$$
\frac{x^{\Delta}}{x}=\frac{1}{r} \frac{r\left(x^{\Delta}\right)^{\gamma}}{x^{\gamma}}\left(\frac{x}{x^{\Delta}}\right)^{\gamma-1} \geq \frac{\alpha_{2}}{r} \frac{\left(r\left(x^{\Delta}\right)^{\gamma}\right)^{\sigma}}{\left(x^{\gamma}\right)^{\sigma}}=\frac{\alpha_{2}}{r}\left(\frac{u-B}{A}\right)^{\sigma} .
$$


So (39) becomes

$$
\begin{aligned}
u^{\Delta} \leq & -A q+B^{\Delta}-A p\left(\frac{\alpha_{2}}{r}\right)^{\gamma}\left[\left(\frac{u-B}{A}\right)^{\sigma}\right]^{\gamma} \\
& +A^{\Delta}\left(\frac{u-B}{A}\right)^{\sigma}-\frac{\gamma A \alpha_{2}}{r}\left[\left(\frac{u-B}{A}\right)^{\sigma}\right]^{2}
\end{aligned}
$$

By (43) and (47), (36) holds. Lemma 7 is proved.

Remark 8. Note that, in general, $\left(x^{\gamma}(t)\right)^{\Delta} \neq\left(x^{\Delta}(t)\right)^{\gamma}$. In [14, (3.6)], it seemed that Şenel had been confused $\left(x^{\gamma}(t)\right)^{\Delta}$ with $\left(x^{\Delta}(t)\right)^{\gamma}$. Some similar mistakes had also been made in [14, (3.8), (3.10)] and, finally, there are some problems about all conclusions of the theorems and corollaries in [14].

\section{Main Results}

In this section we establish Kamenev-type criteria for oscillation of (1). Our approach to oscillation problems of (1) is based largely on the application of the Riccati transformation. Firstly, we give some definitions.

Let $D_{0}=\{s \in \mathbb{T}: s \geq 0\}$ and $D=\left\{(t, s) \in \mathbb{T}^{2}: t \geq s \geq 0\right\}$. For any function $f(t, s): \mathbb{T}^{2} \rightarrow \mathbb{R}$, denote by $f_{2}^{\Delta}$ the partial derivative of $f$ with respect to $s$. Define

$$
\begin{gathered}
(\mathscr{A}, \mathscr{B})=\left\{(A, B): A(s) \in C_{\mathrm{rd}}^{1}\left(D_{0}, \mathbb{R}_{+} \backslash\{0\}\right),\right. \\
\left.B(s) \in C_{\mathrm{rd}}^{1}\left(D_{0}, \mathbb{R}\right), s \in D_{0}\right\} ; \\
\mathscr{H}=\left\{H(t, s) \in C^{1}\left(D, \mathbb{R}_{+}\right): H(t, t)=0,\right. \\
\left.H(t, s)>0, H_{2}^{\Delta}(t, s) \leq 0, t>s \geq 0\right\} .
\end{gathered}
$$

These function classes will be used throughout this paper. Now, we are in a position to give our first theorem.

Theorem 9. Assume that (C1)-(C6) hold and that there exist $(A, B) \in(\mathscr{A}, \mathscr{B})$ and $H \in \mathscr{H}$ such that, for any $t_{1} \in \mathbb{T}$,

$$
\begin{aligned}
& \limsup _{t \rightarrow \infty} \frac{1}{H\left(t, t_{1}\right)} \\
& \times \int_{t_{1}}^{t}\left[H(t, s)\left(A(s) q(s)-B^{\Delta}(s)\right)\right. \\
&\left.\quad-H_{2}^{\Delta}(t, s) B^{\sigma}(s)-\Phi_{1}(s)\right] \Delta s=\infty,
\end{aligned}
$$

where

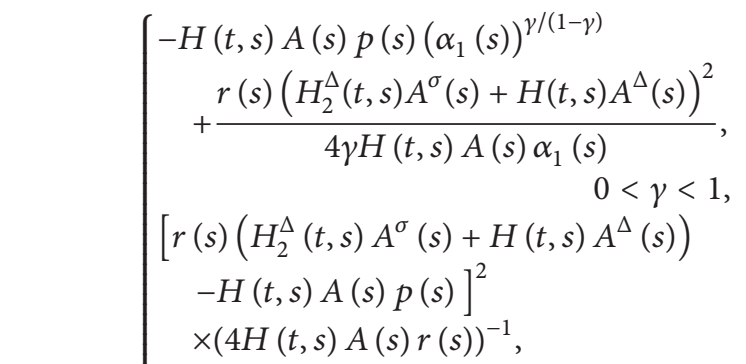

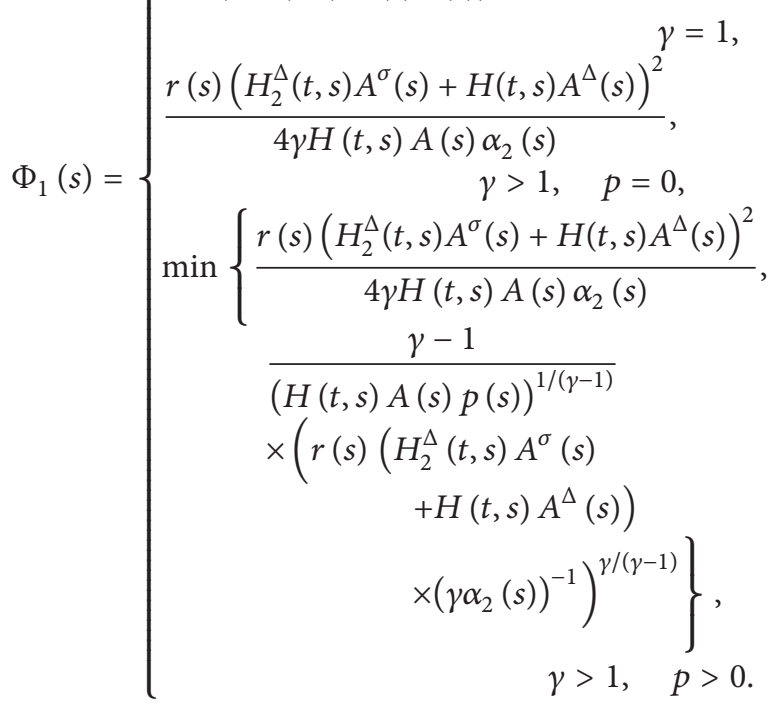

Then, (1) is oscillatory.

Proof. Assume that (1) is not oscillatory. Without loss of generality we may assume that there exists $t_{1} \in\left[t_{0}, \infty\right)_{\mathbb{W}}$ such that $x(t)>0$ for $t \in\left[t_{1}, \infty\right)_{\mathbb{T}}$. Let $u(t)$ be defined by (35). Then, by Lemma 7, (36) holds.

For simplicity, in the following, we let $H_{\sigma}=$ $H(t, \sigma(s)), H=H(t, s), H_{2}^{\Delta}=H_{2}^{\Delta}(t, s)$ and omit the arguments in the integrals.

Multiplying (36), where $t$ is replaced by $s$, by $H$, and integrating it with respect to $s$ from $t_{1}$ to $t$ with $t \in \mathbb{T}$ and $t \geq \sigma\left(t_{1}\right)$, we obtain

$$
\int_{t_{1}}^{t} H\left(A q-B^{\Delta}\right) \Delta s \leq-\int_{t_{1}}^{t} H u^{\Delta} \Delta s+\int_{t_{1}}^{t} H \Phi_{0} \Delta s .
$$

Noting that $H(t, t)=0$, by the integration by parts formula, we have

$$
\begin{aligned}
\int_{t_{1}}^{t} H\left(A q-B^{\Delta}\right) \Delta s \\
\leq H\left(t, t_{1}\right) u\left(t_{1}\right)+\int_{t_{1}}^{t}\left(H_{2}^{\Delta} u^{\sigma}+H \Phi_{0}\right) \Delta s \\
=H\left(t, t_{1}\right) u\left(t_{1}\right)+\int_{t_{1}}^{t} H_{2}^{\Delta} B^{\sigma} \Delta s \\
\quad+\int_{t_{1}}^{t}\left(H_{2}^{\Delta} A^{\sigma}\left(\frac{u-B}{A}\right)^{\sigma}+H \Phi_{0}\right) \Delta s .
\end{aligned}
$$


When $0<\gamma<1$, we have

$$
\begin{aligned}
H_{2}^{\Delta} A^{\sigma}\left(\frac{u-B}{A}\right)^{\sigma}+H \Phi_{0} \\
=-H A p\left(\alpha_{1}\right)^{\gamma /(1-\gamma)}+H_{2}^{\Delta} A^{\sigma}\left(\frac{u-B}{A}\right)^{\sigma} \\
+H A^{\Delta}\left(\frac{u-B}{A}\right)^{\sigma}-\frac{\gamma H A \alpha_{1}}{r}\left[\left(\frac{u-B}{A}\right)^{\sigma}\right]^{2} \\
=-H A p\left(\alpha_{1}\right)^{\gamma /(1-\gamma)}+\frac{r\left(H_{2}^{\Delta} A^{\sigma}+H A^{\Delta}\right)^{2}}{4 \gamma H A \alpha_{1}} \\
\quad-\frac{\gamma H A \alpha_{1}}{r}\left[\left(\frac{u-B}{A}\right)^{\sigma}-\frac{r\left(H_{2}^{\Delta} A^{\sigma}+H A^{\Delta}\right)}{2 \gamma H A \alpha_{1}}\right]^{2} \\
\leq-H A p\left(\alpha_{1}\right)^{\gamma /(1-\gamma)}+\frac{r\left(H_{2}^{\Delta} A^{\sigma}+H A^{\Delta}\right)^{2}}{4 \gamma H A \alpha_{1}} .
\end{aligned}
$$

When $\gamma=1$, we have

$$
\begin{aligned}
& H_{2}^{\Delta} A^{\sigma}\left(\frac{u-B}{A}\right)^{\sigma}+H \Phi_{0} \\
&=-\frac{H A p}{r}\left(\frac{u-B}{A}\right)^{\sigma}+H_{2}^{\Delta} A^{\sigma}\left(\frac{u-B}{A}\right)^{\sigma} \\
&+H A^{\Delta}\left(\frac{u-B}{A}\right)^{\sigma}-\frac{H A}{r}\left[\left(\frac{u-B}{A}\right)^{\sigma}\right]^{2} \\
&= \frac{r\left(H_{2}^{\Delta} A^{\sigma}+H A^{\Delta}\right)-H A p}{r}\left(\frac{u-B}{A}\right)^{\sigma} \\
&-\frac{H A}{r}\left[\left(\frac{u-B}{A}\right)^{\sigma}\right]^{2} \\
&= \frac{\left[r\left(H_{2}^{\Delta} A^{\sigma}+H A^{\Delta}\right)-H A p\right]^{2}}{4 H A r} \\
&-\frac{H A}{r}\left[\left(\frac{u-B}{A}\right)^{\sigma}-\frac{r\left(H_{2}^{\Delta} A^{\sigma}+H A^{\Delta}\right)-H A p}{2 H A}\right]^{2} \\
& \leq \frac{\left[r\left(H_{2}^{\Delta} A^{\sigma}+H A^{\Delta}\right)-H A p\right]^{2}}{4 H A r} .
\end{aligned}
$$

When $\gamma>1$, on the one hand, we have

$$
\begin{aligned}
H_{2}^{\Delta} A^{\sigma}\left(\frac{u-B}{A}\right)^{\sigma}+H \Phi_{0} \\
=-H A p\left(\frac{\alpha_{2}}{r}\right)^{\gamma}\left[\left(\frac{u-B}{A}\right)^{\sigma}\right]^{\gamma} \\
+\left(H_{2}^{\Delta} A^{\sigma}+H A^{\Delta}\right)\left(\frac{u-B}{A}\right)^{\sigma} \\
\quad-\frac{\gamma H A \alpha_{2}}{r}\left[\left(\frac{u-B}{A}\right)^{\sigma}\right]^{2}
\end{aligned}
$$

$$
\begin{aligned}
\leq & \left(H_{2}^{\Delta} A^{\sigma}+H A^{\Delta}\right)\left(\frac{u-B}{A}\right)^{\sigma} \\
& -\frac{\gamma H A \alpha_{2}}{r}\left[\left(\frac{u-B}{A}\right)^{\sigma}\right]^{2} \\
= & \frac{r\left(H_{2}^{\Delta} A^{\sigma}+H A^{\Delta}\right)^{2}}{4 \gamma H A \alpha_{2}} \\
& -\frac{\gamma H A \alpha_{2}}{r}\left[\left(\frac{u-B}{A}\right)^{\sigma}-\frac{r\left(H_{2}^{\Delta} A^{\sigma}+H A^{\Delta}\right)}{2 \gamma H A \alpha_{2}}\right]^{2} \\
\leq & \frac{r\left(H_{2}^{\Delta} A^{\sigma}+H A^{\Delta}\right)^{2}}{4 \gamma H A \alpha_{2}} .
\end{aligned}
$$

On the other hand, when $p>0$, we also have

$$
\begin{aligned}
H_{2}^{\Delta} & A^{\sigma}\left(\frac{u-B}{A}\right)^{\sigma}+H \Phi_{0} \\
= & -H A p\left(\frac{\alpha_{2}}{r}\right)^{\gamma}\left[\left(\frac{u-B}{A}\right)^{\sigma}\right]^{\gamma} \\
& +\left(H_{2}^{\Delta} A^{\sigma}+H A^{\Delta}\right)\left(\frac{u-B}{A}\right)^{\sigma}-\frac{\gamma H A \alpha_{2}}{r}\left[\left(\frac{u-B}{A}\right)^{\sigma}\right]^{2} \\
\leq & -H A p\left(\frac{\alpha_{2}}{r}\right)^{\gamma}\left[\left(\frac{u-B}{A}\right)^{\sigma}\right]^{\gamma} \\
& +\left(H_{2}^{\Delta} A^{\sigma}+H A^{\Delta}\right)\left(\frac{u-B}{A}\right)^{\sigma} .
\end{aligned}
$$

Using the inequality

$$
\lambda a b^{\lambda-1}-a^{\lambda} \leq(\lambda-1) b^{\lambda}
$$

let $\lambda=\gamma$, and

$$
\begin{gathered}
a^{\lambda}=H A p\left(\frac{\alpha_{2}}{r}\right)^{\gamma}\left[\left(\frac{u-B}{A}\right)^{\sigma}\right]^{\gamma}, \\
b^{\lambda-1}=\frac{r\left(H_{2}^{\Delta} A^{\sigma}+H A^{\Delta}\right)}{\gamma \alpha_{2}(H A p)^{1 / \gamma}} .
\end{gathered}
$$

Then we have

$$
\begin{aligned}
& H_{2}^{\Delta} A^{\sigma}\left(\frac{u-B}{A}\right)^{\sigma}+H \Phi_{0} \\
& \quad \leq \frac{\gamma-1}{(H A p)^{1 /(\gamma-1)}}\left(\frac{r\left(H_{2}^{\Delta} A^{\sigma}+H A^{\Delta}\right)}{\gamma \alpha_{2}}\right)^{\gamma /(\gamma-1)} .
\end{aligned}
$$

So, when $p=0$, we get

$$
H_{2}^{\Delta} A^{\sigma}\left(\frac{u-B}{A}\right)^{\sigma}+H \Phi_{0} \leq \frac{r\left(H_{2}^{\Delta} A^{\sigma}+H A^{\Delta}\right)^{2}}{4 \gamma H A \alpha_{2}}
$$


while when $p>0$ we get

$$
\begin{aligned}
H_{2}^{\Delta} A^{\sigma}\left(\frac{u-B}{A}\right)^{\sigma}+H \Phi_{0} & \\
\leq \min & \left\{\frac{r\left(H_{2}^{\Delta} A^{\sigma}+H A^{\Delta}\right)^{2}}{4 \gamma H A \alpha_{2}}, \frac{\gamma-1}{(H A p)^{1 /(\gamma-1)}}\right. \\
& \left.\times\left(\frac{r\left(H_{2}^{\Delta} A^{\sigma}+H A^{\Delta}\right)}{\gamma \alpha_{2}}\right)^{\gamma /(\gamma-1)}\right\} .
\end{aligned}
$$

Therefore, for all $\gamma>0$, by (52), we have

$$
\begin{aligned}
\int_{t_{1}}^{t} H\left(A q-B^{\Delta}\right) \Delta s \leq & H\left(t, t_{1}\right) u\left(t_{1}\right) \\
& +\int_{t_{1}}^{t} H_{2}^{\Delta} B^{\sigma} \Delta s+\int_{t_{1}}^{t} \Phi_{1} \Delta s,
\end{aligned}
$$

which implies that

$$
\int_{t_{1}}^{t}\left[H\left(A q-B^{\Delta}\right)-H_{2}^{\Delta} B^{\sigma}-\Phi_{1}\right] \Delta s \leq H\left(t, t_{1}\right) u\left(t_{1}\right) .
$$

Hence

$$
\frac{1}{H\left(t, t_{1}\right)} \int_{t_{1}}^{t}\left[H\left(A q-B^{\Delta}\right)-H_{2}^{\Delta} B^{\sigma}-\Phi_{1}\right] \Delta s \leq u\left(t_{1}\right)<\infty,
$$

which contradicts (49) and completes the proof.

When $\gamma \geq 1$, let $(A, B)=(1,0)$, and Theorem 9 can be simplified as the following corollary.

Corollary 10. Assume that (C1)-(C6) hold and that there exists $H \in \mathscr{H}$ such that, for any $t_{1} \in \mathbb{T}$,

$$
\limsup _{t \rightarrow \infty} \frac{1}{H\left(t, t_{1}\right)} \int_{t_{1}}^{t}\left[H(t, s) q(s)-\Phi_{2}(s)\right] \Delta s=\infty,
$$

where

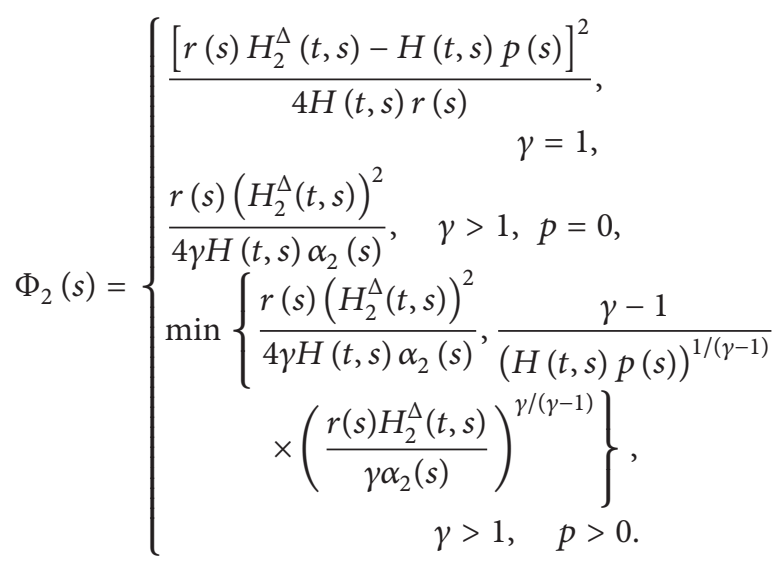

Remark 11. There are some mistakes in [14]. For example, $t^{1 /(\gamma-1)}$ should be $t^{1-\gamma}$ in $[14,(3.22)]$, the symbol $\Delta s$ had not correctly written in $[14,(3.29)], H_{s}^{\Delta}(t, s)+H(t, s) v(s)$ should be $H_{s}^{\Delta}(t, s) z^{\sigma}(s)+H(t, s) v(s)$ in $[14,(3.30),(3.32)]$, and the bracket had been missed in $[14,(3.34)]$.

When $B=0$, (35) is simplified as

$$
u(t)=A(t) \frac{r(t)\left(x^{\Delta}(t)\right)^{\gamma}}{x^{\gamma}(t)}, \quad t \in\left[t_{1}, \infty\right)_{\mathbb{T}} .
$$

Now we have the following theorem.

Theorem 12. Assume that (C1)-(C6) hold and that there exists $A \in C_{\mathrm{rd}}^{1}\left(D_{0}, \mathbb{R}_{+} \backslash\{0\}\right)$ such that, for any $t_{1} \in \mathbb{T}$,

$$
\limsup _{t \rightarrow \infty} \int_{t_{1}}^{t}\left[A(s) q(s)-\Phi_{3}(s)\right] \Delta s=\infty,
$$

where

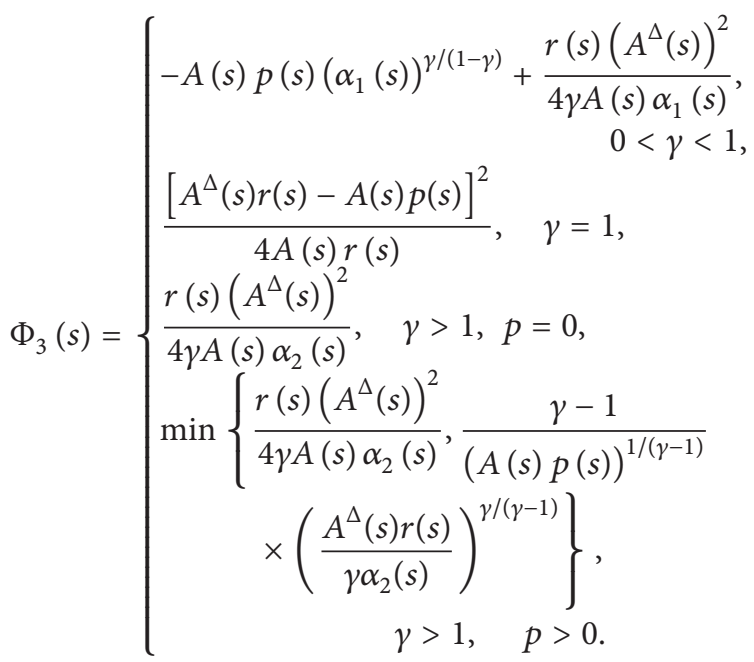

Then, (1) is oscillatory.

Proof. Assume that (1) is not oscillatory. Without loss of generality we may assume that there exists $t_{1} \in\left[t_{0}, \infty\right)_{\mathbb{T}}$ such that $x(t)>0$ for $t \in\left[t_{1}, \infty\right)_{\mathbb{T}}$. Let $u(t)$ be defined by (67). Then, by Lemma 7, we have

$$
u^{\Delta}(t)+A(t) q(t)-\Phi_{0}(t) \leq 0,
$$

where $\Phi_{0}(t)$ is simplified as

$$
\Phi_{0}(t)=\left\{\begin{array}{l}
-A(t) p(t)\left(\alpha_{1}(t)\right)^{\gamma /(1-\gamma)}+A^{\Delta}(t)\left(\frac{u(t)}{A(t)}\right)^{\sigma} \\
-\frac{\gamma A(t) \alpha_{1}(t)}{r(t)}\left[\left(\frac{u(t)}{A(t)}\right)^{\sigma}\right]^{2}, \quad 0<\gamma<1, \\
-A(t) p(t)\left(\frac{\alpha_{2}(t)}{r(t)}\right)^{\gamma}\left[\left(\frac{u(t)}{A(t)}\right)^{\sigma}\right]^{\gamma} \\
+A^{\Delta}(t)\left(\frac{u(t)}{A(t)}\right)^{\sigma}-\frac{\gamma A(t) \alpha_{2}(t)}{r(t)}\left[\left(\frac{u(t)}{A(t)}\right)^{\sigma}\right]^{2}, \\
\gamma \geq 1 .
\end{array}\right.
$$

Then, (1) is oscillatory. 
When $0<\gamma<1$, we have

$$
\begin{aligned}
u^{\Delta} \leq & -A q-A p\left(\alpha_{1}\right)^{\gamma /(1-\gamma)} \\
& +A^{\Delta}\left(\frac{u}{A}\right)^{\sigma}-\frac{\gamma A \alpha_{1}}{r}\left[\left(\frac{u}{A}\right)^{\sigma}\right]^{2} \\
= & -A q-A p\left(\alpha_{1}\right)^{\gamma /(1-\gamma)}+\frac{r\left(A^{\Delta}\right)^{2}}{4 \gamma A \alpha_{1}} \\
& -\frac{\gamma A \alpha_{1}}{r}\left[\left(\frac{u}{A}\right)^{\sigma}-\frac{A^{\Delta} r}{2 \gamma A \alpha_{1}}\right]^{2} \\
\leq & -A q-A p\left(\alpha_{1}\right)^{\gamma /(1-\gamma)}+\frac{r\left(A^{\Delta}\right)^{2}}{4 \gamma A \alpha_{1}} .
\end{aligned}
$$

When $\gamma=1$, we have

$$
\begin{aligned}
u^{\Delta} & \leq-A q+\frac{A^{\Delta} r-A p}{r}\left(\frac{u}{A}\right)^{\sigma}-\frac{A}{r}\left[\left(\frac{u}{A}\right)^{\sigma}\right]^{2} \\
& =-A q+\frac{\left(A^{\Delta} r-A p\right)^{2}}{4 A r}-\frac{A}{r}\left[\left(\frac{u}{A}\right)^{\sigma}-\frac{A^{\Delta} r-A p}{2 A}\right]^{2} \\
& \leq-A q+\frac{\left(A^{\Delta} r-A p\right)^{2}}{4 A r} .
\end{aligned}
$$

When $\gamma>1$, on the one hand, we have

$$
\begin{aligned}
u^{\Delta} & \leq-A q+A^{\Delta}\left(\frac{u}{A}\right)^{\sigma}-\frac{\gamma A \alpha_{2}}{r}\left[\left(\frac{u}{A}\right)^{\sigma}\right]^{2} \\
& =-A q+\frac{r\left(A^{\Delta}\right)^{2}}{4 \gamma A \alpha_{2}}-\frac{\gamma A \alpha_{2}}{r}\left[\left(\frac{u}{A}\right)^{\sigma}-\frac{A^{\Delta} r}{2 \gamma A \alpha_{2}}\right]^{2} \\
& \leq-A q+\frac{r\left(A^{\Delta}\right)^{2}}{4 \gamma A \alpha_{2}}
\end{aligned}
$$

On the other hand, when $p>0$, we also have

$$
u^{\Delta} \leq-A q-A p\left(\frac{\alpha_{2}}{r}\right)^{\gamma}\left[\left(\frac{u}{A}\right)^{\sigma}\right]^{\gamma}+A^{\Delta}\left(\frac{u}{A}\right)^{\sigma} .
$$

Using the inequality

$$
\lambda a b^{\lambda-1}-a^{\lambda} \leq(\lambda-1) b^{\lambda}
$$

let $\lambda=\gamma$ and

$$
a^{\lambda}=A p\left(\frac{\alpha_{2}}{r}\right)^{\gamma}\left[\left(\frac{u}{A}\right)^{\sigma}\right]^{\gamma}, \quad b^{\lambda-1}=\frac{A^{\Delta} r}{\gamma \alpha_{2}(A p)^{1 / \gamma}} .
$$

Then we have

$$
u^{\Delta} \leq-A q+\frac{\gamma-1}{(A p)^{1 /(\gamma-1)}}\left(\frac{A^{\Delta} r}{\gamma \alpha_{2}}\right)^{\gamma /(\gamma-1)} .
$$

So, when $p=0$, we get

$$
u^{\Delta} \leq-A q+\frac{r\left(A^{\Delta}\right)^{2}}{4 \gamma A \alpha_{2}}
$$

while when $p>0$ we get

$$
u^{\Delta} \leq-A q+\min \left\{\frac{r\left(A^{\Delta}\right)^{2}}{4 \gamma A \alpha_{2}}, \frac{\gamma-1}{(A p)^{1 /(\gamma-1)}}\left(\frac{A^{\Delta} r}{\gamma \alpha_{2}}\right)^{\gamma /(\gamma-1)}\right\} .
$$

Therefore, for all $\gamma>0$, we always have

$$
u^{\Delta} \leq-A q+\Phi_{3}
$$

which implies that

$$
A(t) q(t)-\Phi_{3}(t) \leq-u^{\Delta}(t) .
$$

Let $t$ be replaced by $s$, and integrating (82) with respect to $s$ from $t_{1}$ to $t$ with $t \in \mathbb{T}$ and $t \geq \sigma\left(t_{1}\right)$, we obtain

$$
\begin{aligned}
\int_{t_{1}}^{t}\left[A(s) q(s)-\Phi_{3}(s)\right] \Delta s & \leq-\int_{t_{1}}^{t} u^{\Delta}(s) \Delta s=u\left(t_{1}\right)-u(t) \\
& <u\left(t_{1}\right)<\infty
\end{aligned}
$$

which contradicts (68) and completes the proof.

When $\gamma \geq 1$, let $(A, B)=(1,0)$, and Theorem 12 can be simplified as the following corollary.

Corollary 13. Assume that (C1)-(C6) hold and that, for any $t_{1} \in \mathbb{T}$,

$$
\limsup _{t \rightarrow \infty} \int_{t_{1}}^{t}\left[q(s)-\Phi_{4}(s)\right] \Delta s=\infty
$$

where

$$
\Phi_{4}(s)= \begin{cases}\frac{p^{2}(s)}{4 r(s)}, & \gamma=1 \\ 0, & \gamma>1\end{cases}
$$

Then, (1) is oscillatory.

Remark 14. It seems that the inequality in $[14,(3.19)]$ is incorrect. From the definition of $w(t)$ (see $[14,(3.3)]$ ), we can see that $w(t)$ is not always positive and we could not obtain the conclusion in [14, Theorem 3.1]. As a result, we simplify $u(t)$ as (67) which satisfies $u(t)>0$ in this paper and get the correct results.

\section{Examples}

In this section, we will show the application of our oscillation criteria in two examples. We first give an example to demonstrate Theorem 9 (or Corollary 10). 
Example 15. Consider the equation

$$
\left(\left(x^{\Delta}(t)\right)^{\gamma}\right)^{\Delta}+\frac{1}{t}\left(x^{\Delta}(t)\right)^{\gamma}+t(2+\sin t) x^{\gamma}(g(t))=0,
$$

where $r(t)=1, p(t)=1 / t, q(t)=t$, and $\gamma \geq 1$, so we have $-p(t) / r(t)=-1 / t$. Letting $H(t, s)=(t-s)^{2},(A, B)=(1,0)$, we have

(i) $\mathbb{T}=[1, \infty)$, and then there exists $t_{1} \in[1, \infty)$ such that

$$
\begin{gathered}
e_{-p / r}\left(t, t_{0}\right)=e_{-1 / t}(t, 1)=\exp \left(\int_{1}^{t} \xi_{0}\left(-\frac{1}{\tau}\right) d \tau\right) \\
=\exp \left(\int_{1}^{t}\left(-\frac{1}{\tau}\right) d \tau\right)=\frac{1}{t}, \\
\int_{t_{0}}^{\infty}\left(\frac{e_{-p / r}\left(t, t_{0}\right)}{r(t)}\right)^{1 / \gamma} \Delta t=\int_{1}^{\infty}\left(\frac{1}{t}\right)^{1 / \gamma} d t=\infty, \\
\alpha_{2}(t)=\left(r^{1 / \gamma}(t) \int_{t_{1}}^{t} \frac{1}{r^{1 / \gamma}(s)} \Delta s\right)^{\gamma-1} \\
=\left(\int_{t_{1}}^{t} d s\right)^{\gamma-1}=\left(t-t_{1}\right)^{\gamma-1} .
\end{gathered}
$$

When $\gamma=1$, we obtain

$$
\begin{aligned}
\Phi_{2}(s) & =\frac{\left[r(s) H_{2}^{\Delta}(t, s)-H(t, s) p(s)\right]^{2}}{4 H(t, s) r(s)} \\
& =\frac{\left[-2(t-s)-(t-s)^{2} \cdot s^{-1}\right]^{2}}{4(t-s)^{2}}=O(1) .
\end{aligned}
$$

When $\gamma>1$, we obtain

$$
\begin{gathered}
\frac{r(s)\left(H_{2}^{\Delta}(t, s)\right)^{2}}{4 \gamma H(t, s) \alpha_{2}(s)}=\frac{[-2(t-s)]^{2}}{4 \gamma(t-s)^{2}\left(s-t_{0}\right)^{\gamma-1}}=O\left(s^{1-\gamma}\right), \\
\frac{\gamma-1}{(H(t, s) p(s))^{1 /(\gamma-1)}}\left(\frac{r(s) H_{2}^{\Delta}(t, s)}{\gamma \alpha_{2}(s)}\right)^{\gamma /(\gamma-1)} \\
=\frac{\gamma-1}{\left((t-s)^{2} \cdot s^{-1}\right)^{1 /(\gamma-1)}}\left(\frac{-2(t-s)}{\gamma\left(s-t_{0}\right)^{\gamma-1}}\right)^{\gamma /(\gamma-1)} \\
=O\left(s^{1-\gamma}\right)
\end{gathered}
$$

$\Phi_{2}(s)$

$$
\begin{aligned}
& =\min \left\{\frac{r(s)\left(H_{2}^{\Delta}(t, s)\right)^{2}}{4 \gamma H(t, s) \alpha_{2}(s)}, \frac{\gamma-1}{(H(t, s) p(s))^{1 /(\gamma-1)}}\right. \\
& \left.\quad \times\left(\frac{r(s) H_{2}^{\Delta}(t, s)}{\gamma \alpha_{2}(s)}\right)^{\gamma /(\gamma-1)}\right\} \\
& =O\left(s^{1-\gamma}\right) .
\end{aligned}
$$

Hence

$$
\begin{aligned}
& \limsup _{t \rightarrow \infty} \frac{1}{H\left(t, t_{1}\right)} \int_{t_{1}}^{t}\left[H(t, s) q(s)-\Phi_{2}(s)\right] \Delta s \\
& \quad=\limsup _{t \rightarrow \infty} \frac{1}{\left(t-t_{1}\right)^{2}} \int_{t_{1}}^{t}\left[(t-s)^{2} s-\Phi_{2}(s)\right] d s \\
& \quad=\infty .
\end{aligned}
$$

That is, (65) holds. By Corollary 10 we see that (86) is oscillatory.

(ii) $\mathbb{T}=\{n \mid n \geq 2, n \in \mathbb{N}\}$, then there exists $t_{1} \in[2, \infty)_{\mathbb{T}}$ such that

$$
\begin{gathered}
e_{-p / r}\left(t, t_{0}\right)=e_{-1 / t}(t, 2)=\exp \left(\int_{2}^{t} \xi_{1}\left(-\frac{1}{\tau}\right) \Delta \tau\right) \\
=\exp \left(\int_{2}^{t} \log \left(1-\frac{1}{\tau}\right) \Delta \tau\right) \\
=\prod_{k=2}^{t-1}\left(1-\frac{1}{k}\right)=\frac{1}{t-1}, \\
\int_{t_{0}}^{\infty}\left(\frac{e_{-p / r}\left(t, t_{0}\right)}{r(t)}\right)^{1 / \gamma} \Delta t=\int_{2}^{\infty}\left(\frac{1}{t-1}\right)^{1 / \gamma} \Delta t=\infty, \\
\alpha_{2}(t)=\left(r^{1 / \gamma}(t) \int_{t_{1}}^{t} \frac{1}{r^{1 / \gamma}(s)} \Delta s\right)^{\gamma-1}=\left(\int_{t_{1}}^{t} \Delta s\right)^{\gamma-1} \\
=\left(t-t_{1}\right)^{\gamma-1} \cdot
\end{gathered}
$$

Similarly, we can get the conclusion that (86) is oscillatory by Corollary 10.

The second example illustrates Theorem 12.

Example 16. Consider the equation

$$
\left(\frac{1}{t}\left(x^{\Delta}(t)\right)^{\gamma}\right)^{\Delta}+\frac{1}{3 t^{2}}\left(x^{\Delta}(t)\right)^{\gamma}+\frac{1}{t^{7 / 3}} x^{\gamma}(g(t))=0
$$

where $r(t)=1 / t, p(t)=1 / 3 t^{2}, q(t)=1 / t^{7 / 3}=t^{-7 / 3}$, and $\gamma>1 / 3$, so we have $-p(t) / r(t)=-1 / 3 t$. Let $H(t, s)=$ $(t-s)^{2}, A(s)=s^{7 / 3}$, when $\mathbb{T}=[1, \infty)$; then there exists $t_{1} \in[1, \infty)$ such that

$$
\begin{aligned}
& e_{-p / r}\left(t, t_{0}\right) \\
& =e_{-1 / 3 t}(t, 1)=\exp \left(\int_{1}^{t} \xi_{0}\left(-\frac{1}{3 \tau}\right) d \tau\right) \\
& \quad=\exp \left(\int_{1}^{t}\left(-\frac{1}{3 \tau}\right) d \tau\right)=\left(\frac{1}{t}\right)^{1 / 3},
\end{aligned}
$$




$$
\begin{aligned}
\int_{t_{0}}^{\infty} & \left(\frac{e_{-p / r}\left(t, t_{0}\right)}{r(t)}\right)^{1 / \gamma} \Delta t \\
= & \int_{1}^{\infty}\left(\frac{1}{t^{-2 / 3}}\right)^{1 / \gamma} d t \\
= & \int_{1}^{\infty} t^{2 / 3 \gamma} d t=\infty, \\
\alpha_{1}(t) & =\left(\frac{1}{r(t)} \int_{t}^{\infty} q(s) \Delta s\right)^{(1-\gamma) / \gamma} \\
= & \left(t \int_{t}^{\infty} s^{-7 / 3} d s\right)^{(1-\gamma) / \gamma} \\
= & O\left(t^{-(1-\gamma) / 3 \gamma}\right), \\
\alpha_{2}(t) & =\left(r^{1 / \gamma}(t) \int_{t_{1}}^{t} \frac{1}{r^{1 / \gamma}(s)} \Delta s\right)^{\gamma-1} \\
= & \left(\frac{1}{t^{1 / \gamma}} \int_{t_{1}}^{t} s^{1 / \gamma} d s\right)^{\gamma-1} \\
= & O\left(t^{\gamma-1}\right) .
\end{aligned}
$$

When $1 / 3<\gamma<1$, we obtain

$$
\begin{aligned}
\Phi_{3}(s)= & -A(s) p(s)\left(\alpha_{1}(s)\right)^{\gamma /(1-\gamma)}+\frac{r(s)\left(A^{\Delta}(s)\right)^{2}}{4 \gamma A(s) \alpha_{1}(s)} \\
= & -s^{7 / 3} \cdot \frac{1}{3 s^{2}} \cdot s^{-1 / 3} \\
& +\frac{s^{-1} \cdot\left((7 / 3) s^{4 / 3}\right)^{2}}{4 \gamma s^{7 / 3}} \cdot s^{(1-\gamma) / 3 \gamma} \\
= & -1+O\left(s^{1 / 3 \gamma-1}\right) .
\end{aligned}
$$

For $1 / 3<\gamma<1$, we have $-2 / 3<1 / 3 \gamma-1<0$.

When $\gamma=1$, we obtain

$$
\begin{aligned}
\Phi_{3}(s) & =\frac{\left[A^{\Delta}(s) r(s)-A(s) p(s)\right]^{2}}{4 A(s) r(s)} \\
& =\frac{\left((7 / 3) s^{4 / 3} \cdot s^{-1}-s^{7 / 3} \cdot 1 / 3 s^{2}\right)^{2}}{4 s^{7 / 3} \cdot s^{-1}}=O\left(s^{-2 / 3}\right) .
\end{aligned}
$$

When $\gamma>1$, we obtain

$$
\begin{aligned}
& \frac{r(s)\left(A^{\Delta}(s)\right)^{2}}{4 \gamma A(s) \alpha_{2}(s)} \\
& \quad=\frac{s^{-1} \cdot\left((7 / 3) s^{4 / 3}\right)^{2}}{4 \gamma s^{7 / 3} \cdot O\left(s^{\gamma-1}\right)}=O\left(s^{1 / 3-\gamma}\right),
\end{aligned}
$$

$$
\begin{aligned}
& \frac{\gamma-1}{(A(s) p(s))^{1 /(\gamma-1)}}\left(\frac{A^{\Delta}(s) r(s)}{\gamma \alpha_{2}(s)}\right)^{\gamma /(\gamma-1)} \\
& =\frac{\gamma-1}{\left(s^{7 / 3} \cdot 1 / 3 s^{2}\right)^{1 /(\gamma-1)}}\left(\frac{(7 / 3) s^{4 / 3} \cdot s^{-1}}{\gamma \cdot O\left(s^{\gamma-1}\right)}\right)^{\gamma /(\gamma-1)} \\
& =O\left(s^{1 / 3-\gamma}\right) \\
& \Phi_{3}(s) \\
& =\min \left\{\frac{r(s)\left(A^{\Delta}(s)\right)^{2}}{4 \gamma A(s) \alpha_{2}(s)}, \frac{\gamma-1}{(A(s) p(s))^{1 /(\gamma-1)}}\right. \\
& \left.\quad \times\left(\frac{A^{\Delta}(s) r(s)}{\gamma \alpha_{2}(s)}\right)^{\gamma /(\gamma-1)}\right\} \\
& =O\left(s^{1 / 3-\gamma}\right) .
\end{aligned}
$$

For $\gamma>1$, we have $1 / 3-\gamma<-2 / 3<0$.

Hence

$$
\begin{aligned}
& \limsup _{t \rightarrow \infty} \int_{t_{1}}^{t}\left[A(s) q(s)-\Phi_{3}(s)\right] \Delta s \\
& \quad=\limsup _{t \rightarrow \infty} \int_{t_{1}}^{t}\left[s^{7 / 3} \cdot s^{-7 / 3}-\Phi_{3}(s)\right] d s=\infty .
\end{aligned}
$$

That is, (68) holds. By Theorem 12 we see that (92) is oscillatory. Similarly, we can get the same conclusion when $\mathbb{T}=\mathbb{N}$ and $\mathbb{T}=\left\{2^{n}, n \in \mathbb{N}_{0}\right\}$.

\section{Conflict of Interests}

The authors declare that there is no conflict of interests regarding the publication of this paper.

\section{Acknowledgment}

This project was supported by the NNSF of China (no. 11271379).

\section{References}

[1] S. Hilger, Ein maßkettenkalkül mit anwendung auf zentrumsmannigfaltigkeiten [Ph.D. thesis], Universität Würzburg, 1988.

[2] S. Hilger, "Analysis on measure chains-a unified approach to continuous and discrete calculus," Results in Mathematics. Resultate der Mathematik, vol. 18, no. 1-2, pp. 18-56, 1990.

[3] R. P. Agarwal and M. Bohner, "Basic calculus on time scales and some of its applications," Results in Mathematics. Resultate der Mathematik, vol. 35, no. 1-2, pp. 3-22, 1999.

[4] R. P. Agarwal, M. Bohner, D. O'Regan, and A. Peterson, "Dynamic equations on time scales: a survey," Journal of Computational and Applied Mathematics, vol. 141, no. 1-2, pp. 1-26, 2002. 
[5] M. Bohner and A. Peterson, Dynamic Equations on Time Scales: An Introduction with Applications, Birkhäuser, Boston, Mass, USA, 2001.

[6] M. Bohner and A. Peterson, Eds., Advances in Dynamic Equations on Time Scales, Birkhäuser, Boston, Mass, USA, 2003.

[7] A. Del Medico and Q. K. Kong, "Kamenev-type and interval oscillation criteria for second-order linear differential equations on a measure chain," Journal of Mathematical Analysis and Applications, vol. 294, no. 2, pp. 621-643, 2004.

[8] A. Del Medico and Q. K. Kong, "New Kamenev-type oscillation criteria for second-order differential equations on a measure chain," Computers \& Mathematics with Applications, vol. 50, no. 8-9, pp. 1211-1230, 2005.

[9] O. Došlý and S. Hilger, "A necessary and sufficient condition for oscillation of the Sturm-Liouville dynamic equation on time scales," Journal of Computational and Applied Mathematics, vol. 141, no. 1-2, pp. 147-158, 2002.

[10] H. Huang and Q.-R. Wang, "Oscillation of second-order nonlinear dynamic equations on time scales," Dynamic Systems and Applications, vol. 17, no. 3-4, pp. 551-570, 2008.

[11] Y.-C. Qiu and Q.-R. Wang, "Kamenev-type oscillation criteria of second-order nonlinear dynamic equations on time scales," Discrete Dynamics in Nature and Society, vol. 2013, Article ID 315158, 12 pages, 2013.

[12] S. H. Saker, "Oscillation of second-order nonlinear neutral delay dynamic equations on time scales," Journal of Computational and Applied Mathematics, vol. 187, no. 2, pp. 123-141, 2006.

[13] S. H. Saker, R. P. Agarwal, and D. O'Regan, "Oscillation of second-order damped dynamic equations on time scales," Journal of Mathematical Analysis and Applications, vol. 330, no. 2, pp. 1317-1337, 2007.

[14] M. T. Şenel, "Kamenev-type oscillation criteria for the secondorder nonlinear dynamic equations with damping on time scales," Abstract and Applied Analysis, vol. 2012, Article ID 253107, 18 pages, 2012.

[15] Q.-R. Wang, "Oscillation criteria for nonlinear second order damped differential equations," Acta Mathematica Hungarica, vol. 102, no. 1-2, pp. 117-139, 2004.

[16] Q.-R. Wang, "Interval criteria for oscillation of certain second order nonlinear differential equations," Dynamics of Continuous, Discrete \& Impulsive Systems A, vol. 12, no. 6, pp. 769-781, 2005. 


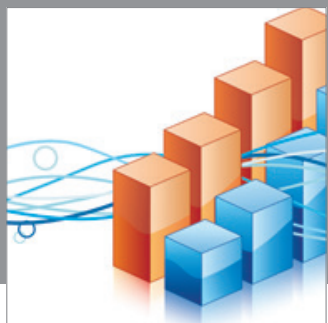

Advances in

Operations Research

mansans

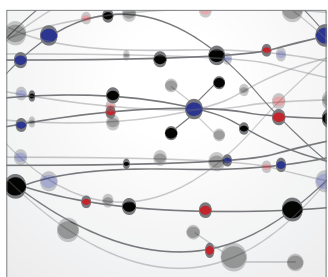

The Scientific World Journal
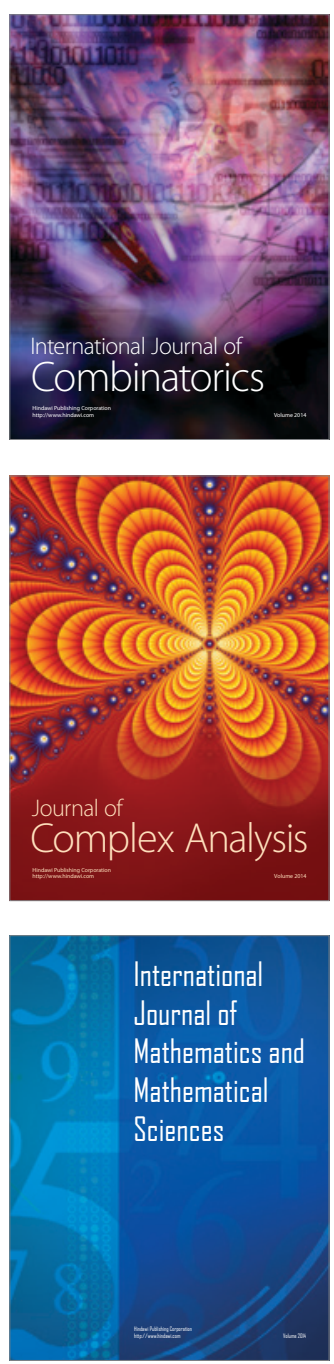
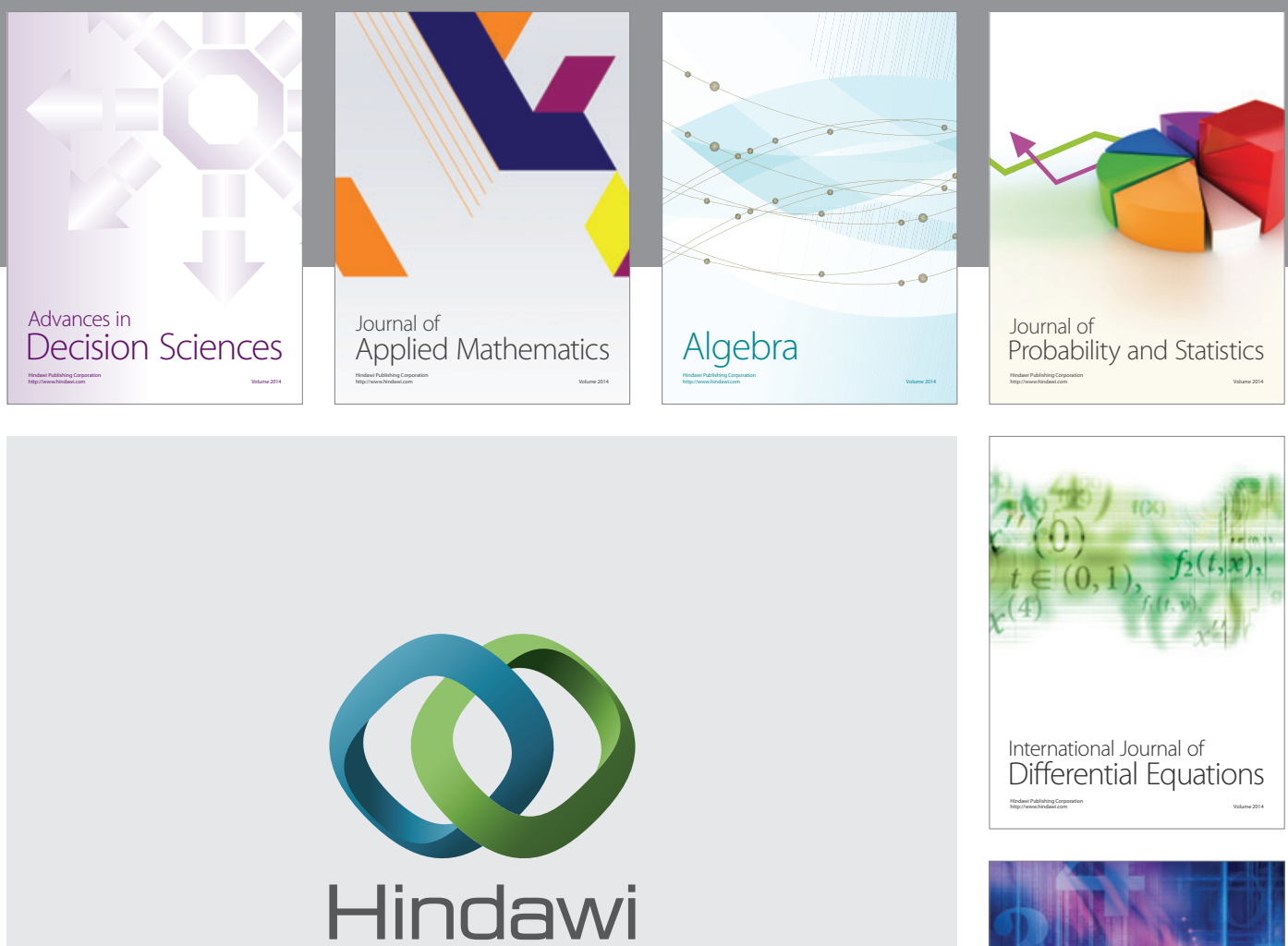

Submit your manuscripts at http://www.hindawi.com
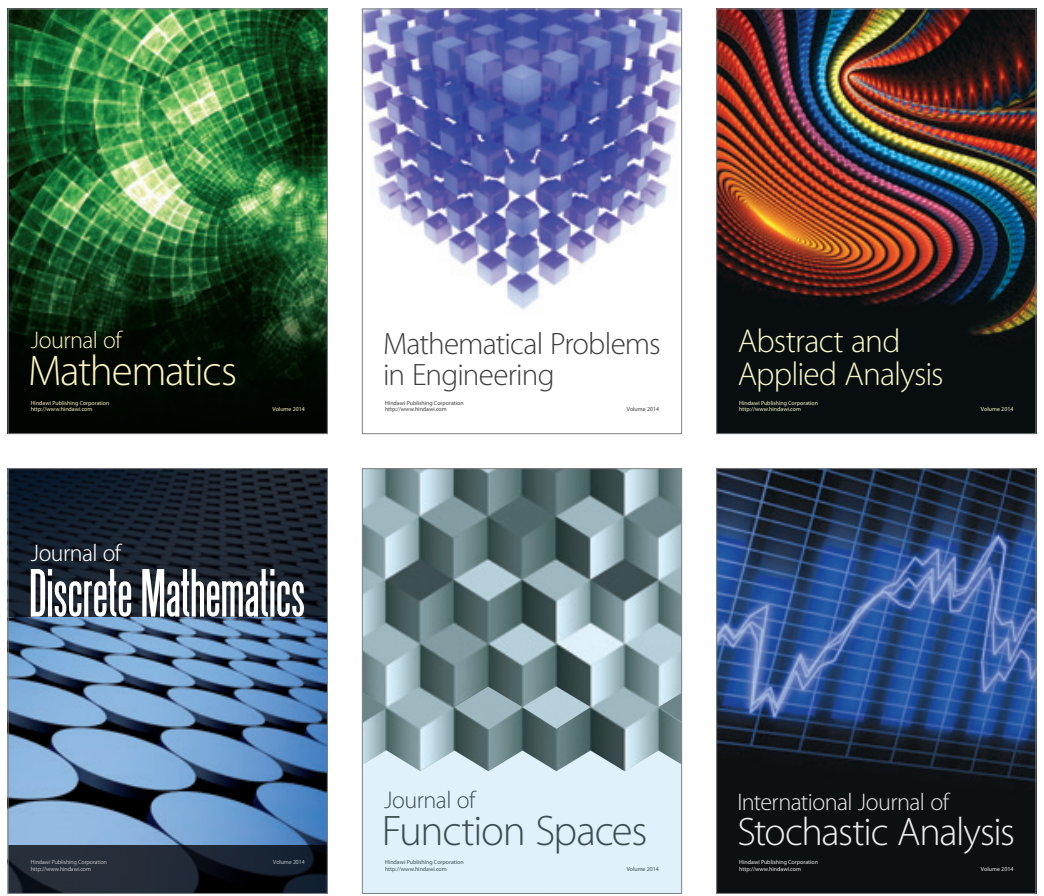

Journal of

Function Spaces

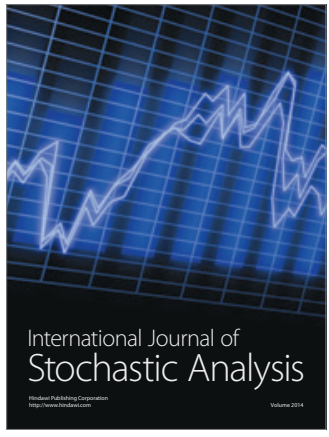

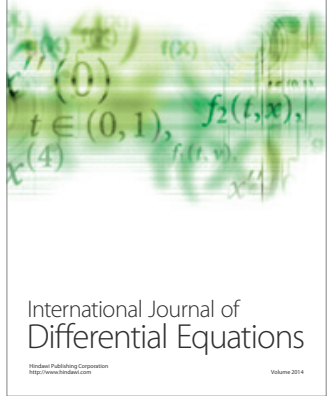
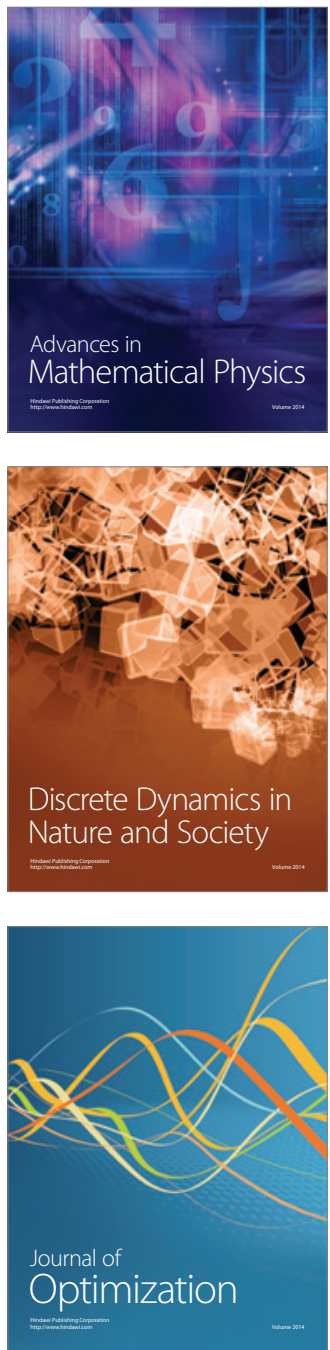